\title{
On the sensitivity of Land Surface Temperature estimates in arid irrigated lands using MODTRAN
}

\author{
J. Rosas , R. Houborg and M. F. McCabe \\ Division of Biological and Environmental Sciences and Engineering, King Abdullah University of Science \\ and Technology, Thuwal, Saudi Arabia \\ Email: jorge.rosas@kaust.edu.sa
}

\begin{abstract}
Land surface temperature (LST) derived from thermal infrared (TIR) satellite data has been reliably used as a remote indicator of evapotranspiration (ET) and surface moisture status. However, in order to retrieve the ET with an accuracy approaching $10 \%$, LST should be retrieved to within $1{ }^{\circ} \mathrm{C}$ or better, disregarding other elements of uncertainty. The removal of atmospheric effects is key towards achieving a precise estimation of LST and it requires detailed information on water vapor. The Thermal Infrared Sensor (TIRS) onboard Landsat 8 captures data in two long wave thermal bands with 100-meter resolution. However, the US Geological Survey has reported a calibration problem of TIRS bands caused by stray light, resulting in a higher bias in one of its two bands (4\% in band 11, $2 \%$ in band 10). Therefore, split-window algorithms for the estimation of LST might not be reliable. Our work will focus on the impact of using different atmospheric profiles (e.g. weather prediction models, satellite) for the estimation of LST derived from MODTRAN by using one of the TIRS bands onboard Landsat 8 (band 10). Sites with in-situ measurements of LST are used as evaluation sources. Comparisons between the measured LST and LST derived based on different atmospheric profile inputs to MODTRAN are carried out from 2 Landsat-overpass days (DOY 153 and 160 2015). Preliminary results show a mean absolute error of around $3{ }^{\circ} \mathrm{C}$ between in-situ and estimated LST over two different crops (alfalfa and carrot) and bare soil.
\end{abstract}

Keywords: Land Surface Temperature, MODTRAN, MODIS, reanalysis, agriculture 
J. Rosas et al., On the sensitivity of Land Surface Temperature estimates in arid irrigated lands ...

\section{INTRODUCTION}

The continuous decline in the fresh water supply is becoming a worldwide problem. Agriculture currently consumes around $70 \%$ of the world's water resources (Gilbert, 2012) and $45 \%$ of the world's food supply comes from irrigated lands (Doell and Siebert, 2002). Moreover, climate change predictions indicate an increase in drought frequency and intensity in arid and semi-arid areas (Stocker et al., 2013). In addition to this, water scarcity will strike most severely the arid and semiarid regions of the world as these have the least buffer in terms of stored water supply. Therefore, our ability to increase the efficiency in the growing of crops will be key towards the adequate management of water resources.

One physical parameter that provides information on water consumption in agriculture is evapotranspiration (ET). ET is responsible for the transfer of large volumes of water (and thus energy) from the soil and vegetation into the atmosphere. ET, along with rainfall and runoff, is one of the most important components of the hydrological cycle as these variables control the availability and distribution of water on the Earth's surface (McCabe and Wood, 2006). Wang et al. (2012) have discussed the importance and challenges in the prediction of hydrological variables associated with the estimation of soil moisture, precipitation, vegetation and water stress, and the linked process of ET in dryland systems. Therefore, the ability to quantify ET in irrigated lands is vital towards the effective management of water resources, particularly in areas of water scarcity.

Land surface temperature is the skin temperature of the Earth's surface and can be derived from thermal infrared remotely sensed data (Weng et al., 2014) and has been reliably used as a remote indicator of ET and surface moisture status. However, in order to retrieve the ET with an accuracy approaching 10\%, LST should be retrieved within $1 \mathrm{~K}$ or better, ignoring other elements of uncertainty [Kustas and Norman (1996); Li et al. (2013)] - is therefore crucial to minimize the errors in the estimation of LST.

Satellite remote sensing provides data on land surface characteristics, useful for mapping land surface energy fluxes, evapotranspiration (Ershadi et al., 2013), soil moisture (McCabe et al., 2005), and vegetation response (Liu et al., 2011). Since 1972, the Landsat satellites have been observing our Earth from space and have been providing information that has helped us understand the state and condition of its surface. The most recent satellite launched is Landsat 8 (February 2013) and has a two-sensor payload: the Operational Land Imager (OLI) and the Thermal Infrared Sensor (TIRS). These two instruments collect image data for nine shortwave bands (15-30 $\mathrm{m}$ resolution) and two longwave bands (100 $\mathrm{m}$ resolution), respectively, at a temporal resolution of 16 days. The TIRS bands (Band 10 and Band 11) collect thermal infrared radiation from 10.6 to $11.19 \mu \mathrm{m}$ and from 11.50 to $12.51 \mu \mathrm{m}$, respectively.

However, the US Geological Survey has reported a calibration problem of TIRS bands caused by stray light, resulting in a higher bias in one of its two bands ( $4 \%$ in band $11,2 \%$ in band 10). Therefore, split-window algorithms for the estimation of LST might not be reliable. In order to avoid imprecise calculations of LST deriving from band 11, other techniques for LST retrieval from band 10, such as atmospheric correction, need to be studied.

The removal of atmospheric effects on the TIR bands is an important step towards achieving a precise estimation of LST (Vidal, 1991) and it requires detailed information on water vapor. The MODIS Atmospheric Profiles Product (MOD 07/MYD 07) provides temperature and moisture profiles, and atmospheric water vapor that could be used as input to the MODTRAN radiative transfer model (Berk et al., 1989) for the atmospheric correction of Landsat imagery. By using MODTRAN, the atmosphere can be modeled as stratified, and its constituent profiles can be defined using the MODIS vertical profiles. Alongside MODIS profiles, atmospheric profiles estimated by reanalysis data could also prove useful towards the removal of atmospheric effects in satellite thermal imagery. In particular, the NCEP/NCAR Reanalysis 1 dataset provides temperature and moisture profiles that can also be input to MODTRAN.

The following work presents some preliminary results on LST derived from Landsat 8 and atmospheric profiles from the MODIS MOD 07 product and NCEP/NCAR Reanalysis 1 data in an irrigated farm in Saudi Arabia. Surface temperature readings from 3 weather stations within the farm will be analyzed alongside the satellite readings in order to understand the underlying surface heterogeneity in satellite retrievals.

\section{METHODOLOGY}

\subsection{Landsat 8 Brightness Temperature}

Landsat provides Level 1 standard data products that have to be converted to actual reflectance and radiance values (USGS, 2013). The Level 1 products consist of quantized and calibrated scaled Digital Numbers (DN) 
J. Rosas et al., On the sensitivity of Land Surface Temperature estimates in arid irrigated lands ...
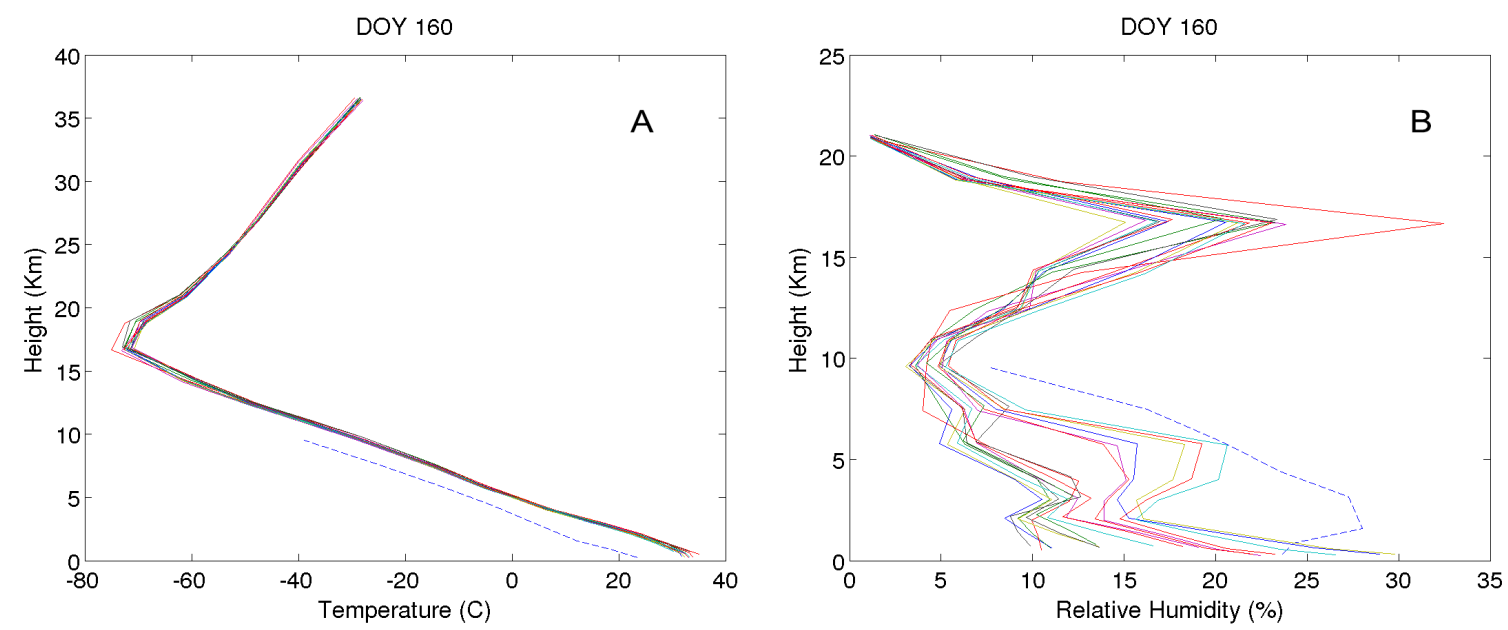

Figure 1. Temperature profiles from MOD 07 (solid lines) and from NCEP/NCAR Reanalysis 1 (dashed line) for the same day DOY 1602015 (A) and relative humidity profiles from MOD 07 (solid lines) and from NCEP/NCAR Reanalysis 1 (dashed line) for the same day (B). Temperatures in degrees Celsius.

representing image data. The conversion to Top of the Atmosphere (TOA) Radiance can be achieved by using the radiance rescaling factors provided in the metadata file (USGS, 2013):

$$
L_{\lambda}=M_{L} Q_{c a l}+A_{L}
$$

where:

$L_{\lambda}$ is the TOA spectral radiance $\left[W /\left(m^{2} \cdot \operatorname{srad} \cdot \mu m\right)\right]$,

$M_{L}$ is the band-specific multiplicative rescaling factor from the metadata (RADIANCE_MULT_BAND_X, where $\mathrm{X}$ is the band number),

$A_{L}$ is the band-specific additive rescaling factor from the metadata (RADIANCE_ADD_BAND_X, where X is the band number),

$Q_{c a l}$ is the quantized and calibrated standard product pixel values (DN).

The TIRS band data can be converted to brightness temperature following Eq. 1 and using the thermal constants provided in the metadata file:

$$
T=\frac{K_{2}}{\ln \left(\frac{K_{1}}{L_{\lambda}}+1\right)}
$$

where:

$T$ is the at-satellite brightness temperature (in Kelvin,)

$L_{\lambda}$ is the TOA spectral radiance $\left[W /\left(m^{2} \cdot \operatorname{srad} \cdot \mu m\right)\right]$,

$K_{1}$ and $K_{2}$ are the band-specific thermal conversion constant from the metadata (K1/K2_CONSTANT_BAND_X, where $\mathrm{x}$ is the band number).

\subsection{MODTRAN}

MODTRAN (Berk et al., 1989) is an atmospheric radiative transfer model that serves as a standard atmospheric band model for remote sensing. The atmospheric profiles from the MOD 07 product and the NCEP/NCAR Reanalysis data were introduced into MODTRAN as vertical profiles (Fig. 1).

The MOD 07 product is available at a $1-k m$ pixel resolution, while the NCEP/NCAR Reanalysis data at a 2.5 degree resolution. In order to speed-up the processing of LST derived from the MOD 07 product, the MOD 07 profile information was divided into 25 regions, and the average of each region (Fig.1) was used as the atmospheric profile input to MODTRAN. Since the Landsat scene covers an area of $170 \mathrm{~km} \times 185 \mathrm{~km}$, each MOD 07 averaged profile covers approximately $1200 \mathrm{~km}^{2}$. 
J. Rosas et al., On the sensitivity of Land Surface Temperature estimates in arid irrigated lands ...

By modelling the atmosphere with MODTRAN, the radiances emitted by the surface of the Earth can be estimated if the radiances at the sensor (satellite) are known. The sensor radiance $L S_{j}$ can be expressed as (Schmugge et al., 1998):

$$
L S_{j}=\left[\epsilon_{j} L_{j}^{B B}(T)+\left(1-\epsilon_{j}\right) L_{j}^{s k y}\right] \tau_{j}+L_{j}^{a t m}
$$

where,

$\epsilon_{j}$ is the surface emissivity at wavelength $j$,

$L_{j}^{B B}(T)$ is the spectral radiance from a blackbody at surface temperature $\mathrm{T}$,

$L_{j}^{s k y}$ is the spectral radiance incident upon the surface from the atmosphere, calculated from MODTRAN,

$L_{j}^{a t m}$ is the spectral radiance emitted by the atmosphere, calculated from MODTRAN,

$\tau_{j}$ is the spectral atmospheric transmission, from MODTRAN,

$L S_{j}$ is the spectral radiance observed by the sensor.

Emissivity was calculated based on the NDVI Thresholds Method developed by Sobrino et al. (2004), which obtains the emissivity values from the NDVI considering three different cases:

1. NDVI $<0.2$ : In this case, the pixel is considered as bare soil and the emissivity is assumed to be 0.96 .

2. NDVI $>0.5$ : NDVI values larger than 0.5 are considered as fully vegetated and the emissivity is assumed to be 0.98 .

3. $0.2 \leq \mathrm{NDVI} \leq 0.5$ : NDVI values between (or equal to) 0.2 and 0.5 are considered as being a mixture of vegetation and soil pixels and the emissivity is calculated as follows:

$$
\epsilon=\epsilon_{v} P_{v}+\epsilon_{s}\left(1-P_{v}\right)
$$

where $\epsilon_{v}$ is the vegetation emissivity (0.98) and $\epsilon_{s}$ is the soil emissivity (0.96), $P_{v}$ is the vegetation proportion:

$$
P_{v}=\left[\frac{\mathrm{NDVI}_{-} \mathrm{NDVI}_{\text {min }}}{\mathrm{NDVI}_{\max }-\mathrm{NDVI}_{\min }}\right]^{2}
$$

Using the atmospheric radiative transfer model MODTRAN (Berk et al., 1989), the radiances emitted by the surface can be estimated using the radiances at the sensor data using Eq.3. If band emissivity is known, it is possible to correct for the reflected sky radiation in Eq.3 and the surface temperature can be calculated by inversion of the Plancks Law (Li et al., 2004):

$$
T_{s}=\frac{k_{2}}{\ln \left[\frac{k_{1}}{I_{\lambda}^{B}}+1\right]}
$$

where,

$T_{s}$ is the surface temperature in Kelvin,

$I_{\lambda}^{B}$ is the band radiance (from Eq.3, $\epsilon_{j} L_{j}^{B B}(T)$ ),

$k_{1}$ and $k_{2}$ are calibration constants chosen to optimize the approximation for the band pass of the sensor $\left(k_{1}=774.89, k_{2}=1321.08\right.$ for Landsat 8$)$.

\section{RESULTS}

The atmospheric profiles derived from the MOD07 product and the NCEP/NCAR Reanalysis 1 (Fig.1 A) show similar values for the temperature profiles, although the NCEP/NCAR Reanalysis 1 is underestimating the values from the MOD07 product. As for the relative humidity, the NCEP/NCAR Reanalysis 1 seems to be estimating different values from the MOD07 product during the closest $5 \mathrm{~km}$ in height to the Earth's surface (Fig.1 B). The MOD07 and NCEP/Reanalysis 1 seem to be estimating the trend of relative humidity accurately from 6 to $10 \mathrm{~km}$, although with differences between their absolute values. 


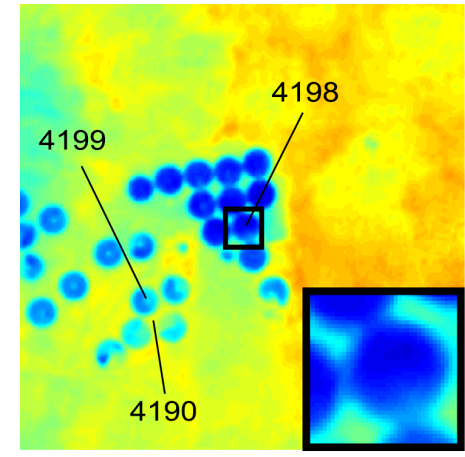

A

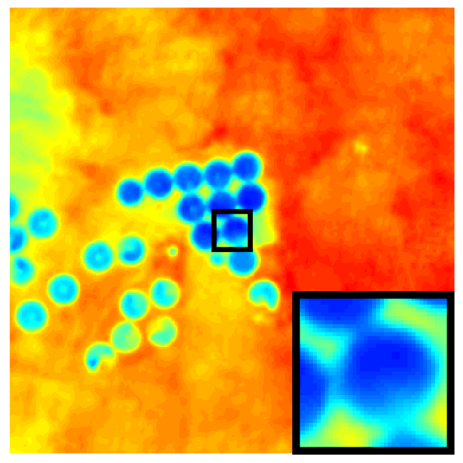

B

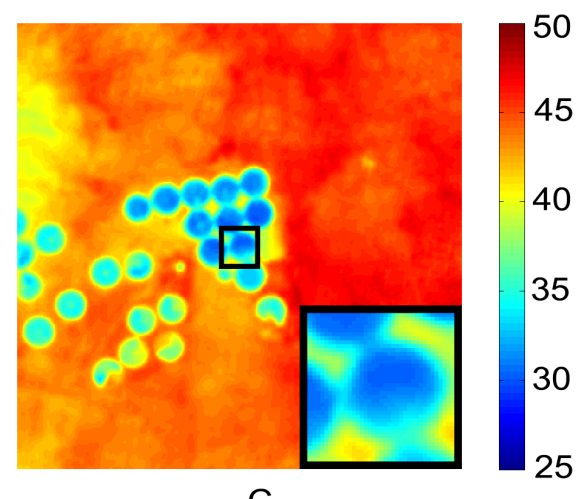

C

Figure 2. (A) Brightness temperature from Landsat in the Tawdeehiya farm (Al-Kharj, Saudi Arabia) for DOY 160 2015, (B) LST derived from MODTRAN using the MOD 07 atmospheric profile and (C) the NCEP/NCAR Reanalysis 1 profile for the same day. All temperatures in degrees Celsius.

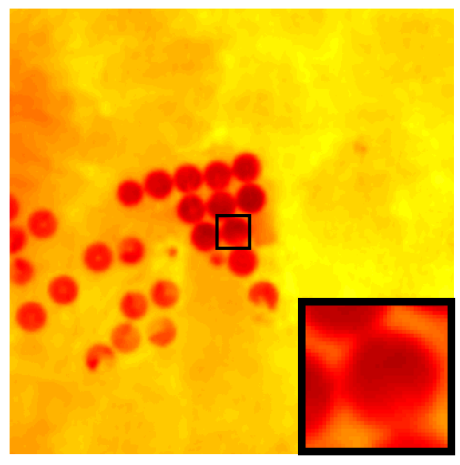

A

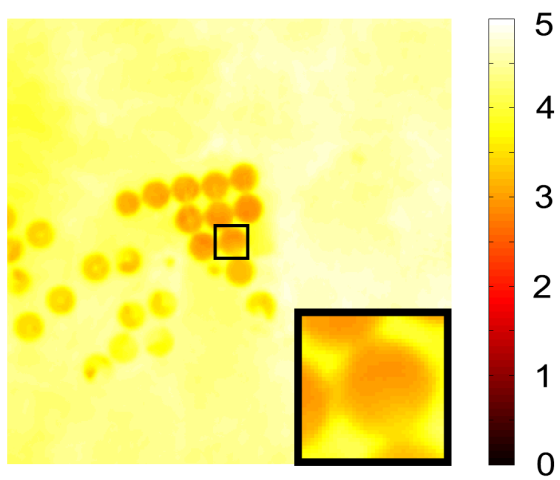

B

Figure 3. (A) LST using the MOD 07 profile minus Landsat brightness temperature and (B) NCEP/NCAR Reanalysis LST minus Landsat brightness temperature for DOY 160 2015. Temperature differences in degrees Celsius.
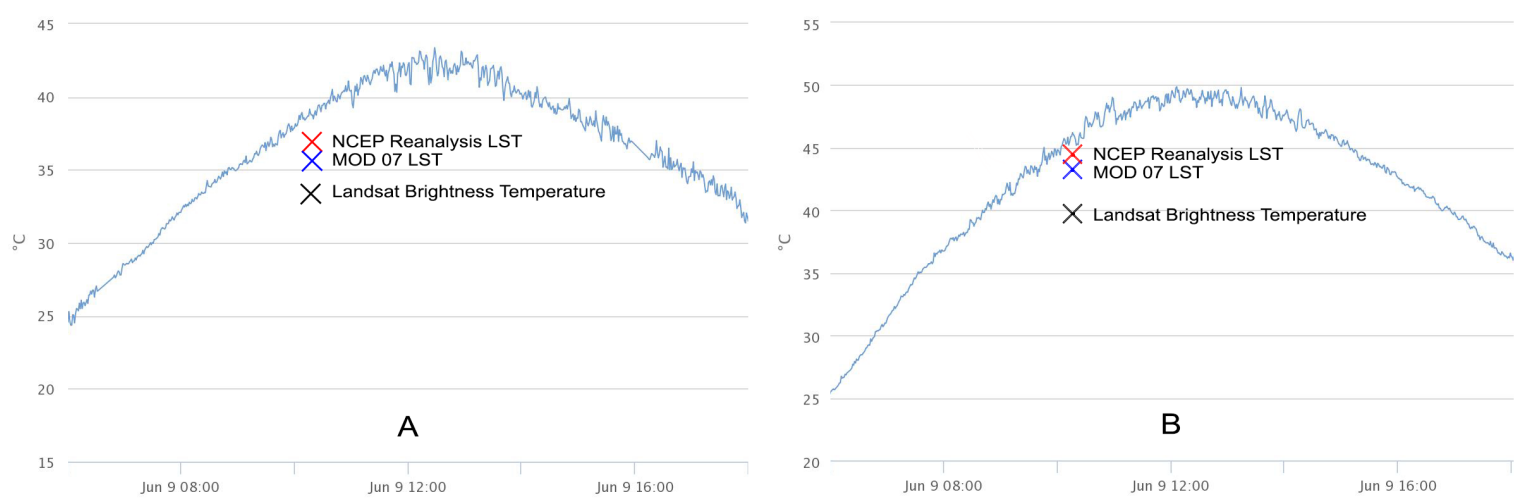

Figure 4. Temperature profile from Station 4199 (inside a carrot field) for DOY 1602015 (A) and from Station 4190 (between 3 fields) for the same day (B). Landsat brightness temperature (black), MOD 07 LST (blue), and NCEP Reanalysis LST (red) are also shown. Temperature in degrees Celsius. 
Preliminary results of LST derived from MODTRAN and atmospheric profiles for the Tawdeehiya farm in the Al-Kharj region of Saudi Arabia are shown in Fig.2. The location of the 3 weather stations currently installed in the farm can be seen in Fig.2 A. Station 4198 and Station 4199 are located over alfalfa and carrots, respectively, while Station 4190 is located over soil. Temperature differences between the soil and the irrigated areas can reach large values, ranging from 15 to $20{ }^{\circ} \mathrm{C}$. In-situ measurements for DOY 153 and 160 in 2015 show a mean absolute error of $2.9^{\circ} \mathrm{C}$ for LST derived from the MOD 07 product, and $3.0^{\circ} \mathrm{C}$ for LST derived from the NCEP/NCAR Reanalysis 1 data. Fig.2 also shows a subset of a Landsat 8 scene for DOY 160 in 2015. Fig.2 A shows the brightness temperature (at-satellite temperature). Fig. 2 B and Fig.2 C show the LST derived from the MOD 07 profile and the LST derived from the NCEP/NCAR Reanalysis 1 profile for the same day, respectively.

Fig.3 A shows the absolute difference between the LST calculated from the MOD07 profile and Landsat brightness temperature, while Fig.3 B shows the absolute difference between the LST calculated from the NCEP/NCAR Reanalysis 1 data and Landsat brightness temperature for the same day. Larger temperature differences can be seen between the NCEP/NCAR Reanalysis 1 LST and the original Landsat brightness temperature. The effect of emissivity values on the estimation of LST can also be seen in Fig.3, as the largest differences in temperature can be observed in areas without crops.

Fig. 4 shows the temperature profiles of weather stations installed inside (Station 4199) and outside (Station 4190) a carrot field, respectively. The temperature profiles from the weather stations (Fig.4) show larger values than the estimated temperature from MOD07 and NCEP/NCAR Reanalysis. In other words, LST from the soil and crops is being underestimated by both approaches.

\section{SUMMARY AND CONCLUSIONS}

ET is one of the most important components of the hydrological cycle and its effective estimation depends on the accuracy of LST calculations, and with that, the correct modelling of the atmosphere composition. Reliable Landsat 8 LST estimation is a needed research topic that this work is trying to address. Surface temperature has proven to be a viable calibration variable to improve model estimation of linked variables, such as ET [McCabe et al. (2005); Stisen et al. (2011)]. The preliminary results previously shown suggest that further work is needed in order to try to reduce the current errors in the estimation of LST: atmospheric profiles from MOD 07 do not extend to the surface of the Earth (Fig.1), while the profiles from NCEP/NCAR Reanalysis are limited to $300 \mathrm{mb}$ (or $10 \mathrm{~km}$ height approx., Fig. 1). In addition to this, there is not enough in-situ data to draw definitive conclusions on the performance of both methods. The high temperature differences between bare soil and farmland during the summer also represent a challenge in the estimation of LST by remote sensing, as the sensor on-board the satellite could capture information from different signals into a single pixel. Moreover, variations of subpixel surface geometry and shadows from the canopy are generally present, resulting in emissivity and temperature variations that are combined into a single pixel (McCabe et al., 2008). These variations can interact in nonlinear ways, as the combined radiance depends on the surface materials and on the temperature distributions of each of those materials. Therefore, future work will consist on the addition of other sources of atmospheric profiles, such as AIRS on-board the AQUA satellite, as well as the use of thermal information acquired by unmanned aerial vehicles (UAVs) in order to understand the sub-pixel variability within each Landsat pixel. Adding Landsat 8 to the list of high-resolution TIR-capable satellites will enhance the temporal availability of LST.

\section{ACKNOWLEDGEMENT}

Research reported in this publication was supported by the King Abdullah University of Science and Technology (KAUST). We greatly appreciate the logistical, equipment and scientific support offered to our team by Mr Jack King, Mr Alan King and employees of the Tawdeehiya Farm in Al Kharj, Saudi Arabia, without whom this research would not have been possible.

\section{REFERENCES}

Berk, A., L. S. Bernstein, and D. C. Robertson (1989). Modtran: A moderate resolution model for lowtran.

Doell, P. and S. Siebert (2002). Global modeling of irrigation water requirements. Water Resources Research 38(4), 8-1-8-10.

Ershadi, A., M. McCabe, J. Evans, and J. Walker (2013). Effects of spatial aggregation on the multi-scale estimation of evapotranspiration. Remote Sensing of Environment 131(0), 51 - 62. 
J. Rosas et al., On the sensitivity of Land Surface Temperature estimates in arid irrigated lands ...

Gilbert, N. (2012). Water under pressure. Nature 483(7389), 256-257. cited By 12.

Kustas, W. P. and J. M. Norman (1996). Use of remote sensing for evapotranspiration monitoring over land surfaces. Hydrological Sciences Journal 41(4), 495-516.

Li, F., T. J. Jackson, W. P. Kustas, T. J. Schmugge, A. N. French, M. H. Cosh, and R. Bindlish (2004). Deriving land surface temperature from landsat 5 and 7 during smex02/smacex. Remote Sensing of Environment 92(4), 521-534.

Li, Z.-L., B.-H. Tang, H. Wu, H. Ren, G. Yan, Z. Wan, I. F. Trigo, and J. A. Sobrino (2013). Satellite-derived land surface temperature: Current status and perspectives. Remote Sensing of Environment 131(0), 14-37.

Liu, Y. Y., R. A. M. de Jeu, M. F. McCabe, J. P. Evans, and A. I. J. M. van Dijk (2011). Global long-term passive microwave satellite-based retrievals of vegetation optical depth. Geophysical Research Letters 38(18), n/an/a. L18402.

McCabe, M. F., L. K. Balick, J. Theiler, A. R. Gillespie, and A. Mushkin (2008). Linear mixing in thermal infrared temperature retrieval. International Journal of Remote Sensing 29(17-18), 5047-5061.

McCabe, M. F., J. D. Kalma, and S. W. Franks (2005). Spatial and temporal patterns of land surface fluxes from remotely sensed surface temperatures within an uncertainty modelling framework. Hydrology and Earth System Sciences 9(5), 467-480.

McCabe, M. F. and E. F. Wood (2006). Scale influences on the remote estimation of evapotranspiration using multiple satellite sensors. Remote Sensing of Environment 105(4), 271 - 285.

McCabe, M. F., E. F. Wood, and H. Gao (2005). Initial soil moisture retrievals from amsr-e: Multiscale comparison using in situ data and rainfall patterns over iowa. Geophysical Research Letters 32(6). L06403.

Schmugge, T., S. J. Hook, and C. Coll (1998). Recovering surface temperature and emissivity from thermal infrared multispectral data. Remote Sensing of Environment 65(2), 121-131.

Sobrino, J. A., J. C. Jimnez-Muoz, and L. Paolini (2004). Land surface temperature retrieval from landsat tm 5. Remote Sensing of Environment 90(4), 434-440.

Stisen, S., M. F. McCabe, J. C. Refsgaard, S. Lerer, and M. B. Butts (2011). Model parameter analysis using remotely sensed pattern information in a multi-constraint framework. Journal of Hydrology 409(12), 337 349.

Stocker, T., D. Qin, G. Plattner, M. Tignor, S. Allen, J. Boschung, A. Nauels, Y. Xia, V. Bex, and P. Midgley (2013). Ipcc climate change 2013: The physical science basis. contribution of working group i to the fifth assessment report of the intergovernmental panel on climate change. Cambridge University Press.

USGS (2013). Using the Landsat 8 Product http://landsat.usgs.gov/. Accessed: 2015-04-27.

Vidal, A. (1991). Atmospheric and emissivity correction of land surface temperature measured from satellite using ground measurements or satellite data. International Journal of Remote Sensing 12(12), 2449-2460.

Wang, L., P. D’Odorico, J. P. Evans, D. J. Eldridge, M. F. McCabe, K. K. Caylor, and E. G. King (2012). Dryland ecohydrology and climate change: critical issues and technical advances. Hydrology and Earth System Sciences 16(8), 2585-2603.

Weng, Q., P. Fu, and F. Gao (2014). Generating daily land surface temperature at landsat resolution by fusing landsat and \{MODIS\} data. Remote Sensing of Environment 145(0), 55 - 67. 